not clear. Gradual withdrawal of corticosteroids after a short course of treatment is probably a sensible precaution. If corticosteroids are contraindicated or produce unwanted side effects, other immunosuppressive agents may be effective. ${ }^{19}$

Adam D Timmis

Senior Registrar in Cardiology,

Guy's Hospital,

London SE1 9RT

1 Dressler $W$. A complication of myocardial infarction resembling idiopathic, recurrent benign pericarditis [Abstract]. Circulation 1955;12:697.

Soloff LA, Zatuchni J, Janton H, O'Neill TJ, Glover RP. Reactivation of rheumatic fever following mitral commissurotomy. Circulation 1953;8:481-4.

Dresser W The post-myocardial-infarction syndrome. Arch Intern Med 1959;103:28-42.

4 Dressler $W$. Flare-up of pericarditis complicating myocardial infarction after two years of steroid herapy. Am Heart f 1959;57:501-6.

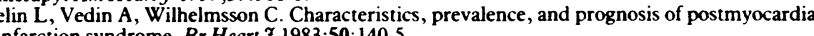
infarction syndrome. Br Hearl $f$ 1983;50:140-5.

1968;183:393-5.

Toole JC, Silverman ME. Pericarditis of acute myocardial infarction. Chest 1975;67:647-53.

Thadani U, Chopra MP, Aber CP, Portal RW. Pericarditis after acute myocardial infarction. Br Med f 1971;ii:135-7.

Bulkley BH, Roberts WC. Steroid therapy during acute myocardial infarction: a cause of delayed healing and of ventricular aneurysm. Am $\mathcal{J}$ Med 1974;56:244-50

Lessof MH. Immunological reactions in heart disease. Br Heart $\mathcal{Z}$ 1978; 40:211-4.

Van Der Gold $\mathrm{H}$. Anti-heart antibodies in the postpericardiotomy and the postmyocardial infarction syndromes. Lancet 1964;ii:617-2

2 Robinson J, Brigden W. Recurrent pericarditis. Br Med $\mathcal{f} 1968$;ii 272-5.

3 Kuch J, Chorzelski T. Immunofluorescence studies in recent myocardial infarction. Cardiovas

14 Liem KL, Ten Veen JH, Lie KI, Feltkamp TEW, Durrer D. Incidence and significance of heart muscle antibodies in patients with acute myocardial infarction and unstable angina. Acta Med

15 Williams RKT, Nagle RE, Thompson RA. Postcoronary pain and the postmyocardial infarction syndrome. Br Heart f 1984;51:327-9.

6 Itoh $\mathrm{K}$, Ohkuni H, Kimura E. Kimura Y. Immunoserological studies on myocardial infarction and postmyocardial infarction syndrome. $f_{p n} \mathrm{Heart} \mathcal{f}$ 1969;10:485-502.

17 Trueman T, Thompson RA, Cummins P, Littler WA. Heart antibodies in cardiomyopathies. $\mathrm{Br}$ Heart $\mathcal{F}$ 1981;46:296-301.

18 Lawrence MSTA, Wright $R$. Tamponade in Dressler's syndrome with immunological studies. $B r$ Med J 1972;i:665-6.

9 Kossowsky WA, Kim KS, Tobin MS. Antimetabolite therapy in the post-myocardial-infarction syndrome. Am Heart f 1972;83:527-31.

\section{Drugs and intravenous fluids}

In 1976 the Department of Health issued a circular (HC76(9)) commending to health authorities the report of a working party on adding drugs to intravenous infusion fluids. ' To those who are not concerned with the emergency treatment of patients this may appear a trivial and even slightly abstruse problem-but this impression would not be correct. It was then, and continues to be, a matter of concern to many doctors, nurses, pharmacists, and financial administrators-and one in which scant progress has been made in the past eight years.

Drugs are added to between a third and almost a half of all intravenous infusions set up in hospital wards. The drugs most frequently given in this way are potassium, antibiotics, and antiarrhythmics. In addition, many drugs are given intermittently intravenously through the giving set of the infusion. Doctors prescribing drugs to be given in this way do not always appreciate the potential problems-for example, asepsis cannot always be maintained and the drug may interact with the intravenous fluid, with reduced drug efficacy or increased drug toxicity. There is even a risk that the drug may not get to the patient at all (p 678).

Several factors contribute to the unsatisfactory administration of drugs by the intravenous route, as the 1976 circular points out. Firstly, most of the information about the administration of drugs and possible drug interactions is published in the pharmaceutical journals. Since two thirds of infusions are set up after $5 \mathrm{pm}$-and pharmacists usually work a standard eight hour day - the doctor may not have easy access to the facts that he needs. Secondly, the responsibility for adding drugs to the infusion fluids frequently rests with the nurse. Too often her authority, responsibility, and training are either not defined or are inadequate and local policies and standards of competence may vary widely. Thirdly, the safety of intravenous drug treatment may be prejudiced because prescriptions are inaccurate or the instructions are not given clearly, or both.

At a recent meeting in Cambridge members of the medical, nursing, and pharmaceutical professions discussed how far these difficulties had been overcome. Sadly, few changes appear to have taken place, and none of the professions emerge with great credit. Thus few medical schools include specific undergraduate teaching on the intravenous administration of drugs. Another useful measure would be to draw up a shortlist of rules to give to all doctors likely to administer drugs intravenously, so that the common problems could be recognised and avoided. For example, drugs should not be mixed in a syringe unless the data sheet, or other reliable source of information, indicates that this is permissible; drugs should be added to infusions only immediately before use; no more than one drug should be added to a minipack and no more than two to an infusion pack (again checking that they are compatible both with each other and with the infusion fluid). A new book, Guide to Parenteral Administration of Drugs, provides a good source of information, for it outlines the principles underlying the intravenous administration of drugs and gives details of most of the drugs that are given parenterally. ${ }^{2}$ It also gives information about the route of administration and which drug and fluid combinations are incompatible.

New house physicians and surgeons often regard the intravenous line as just a convenient route for the administration of drugs. Their attitude might change, however, if part of the introductory course, which most hospitals offer their new resident staff, was devoted to a discussion of the difficulties that may arise when giving drugs by this route. Teaching might be taken by a clinician with a special interest (perhaps an anaesthetist) together with a pharmacist or a nurse, or both. These sessions should build on the basic information given to undergraduates.

Possibly it is the pharmacists who should be providing a clear lead, and in some hospitals this may be happening. In most hospitals, however, pharmacists do not provide a 24 hour service for making up infusions, or even for giving advice, and in this respect the role of the pharmacist (like that of the doctor) has altered little since 1976. Nurses are the one group who have attempted to make changes, and-given that they usually administer this form of treatment-have set about providing full specialist training for some of their staff. Unfortunately, these efforts have been sporadic and no national standards have been agreed; furthermore, nursing education on all aspects of therapeutics remains limited. This will have to change if the responsibility for intravenous treatment is to pass into their hands - a move which is not only possible but probably desirable, given the medical profession's longstanding reluctance to take the problem seriously.

Since 1976 drug and therapeutic committees have been set up to coordinate, improve, and simplify drug treatment in hospital. Yet few of these committees appear to have considered the problem of drugs and intravenous therapy, and where overall authority lies for this method of drug treatment is still not clear. Technical advances have occurred, however, and the equipment used to administer 
drugs intravenously has also improved. Some manufacturers now provide some drugs in fluids ready for infusion. These preparations may have advantages, but we must be wary of such a change in therapeutic practice that is led by industry.

Perhaps the first move should be to obtain more information about whether the therapeutic scene is really as black as the article in this week's $B M \mathcal{F}$ suggests. If it is, the Department of Health should re-examine the problem.

A BRECKENRIDGE

Professor of Clinical Pharmacology, University of Liverpool,

Liverpool L69 3BX

A M J WOOLFSON

Consultant in Clinical Chemistry, City Hospital,

Nottingham NG5 IPB

I Department of Health and Social Security. Report if the working party on the addition of drugs to intravenous infusion fluids. London: DHSS, 1976. HC76 9

Hipwell CE, Mashford ML, Robertson MB. Guide to parenteral administration of drugs. Sydney, Australia: ADIS Press, 1984.

\section{Common bacterial pathogens and resistance to antibiotics}

Bacteria have successfully survived the antibiotic era, and their mechanisms of resistance to antibacterials are better understood.' So how extensive is resistance among the common pathogens at present, and what can be done to guard the future? Much of the relevant information comes from North America, but variations in trends are seen throughout the world-and, indeed, geographical differences in resistance rates may be large.

Nearly all strains of Staphylococcus aureus are sensitive to cloxacillin and other $\beta$ lactamase stable penicillins, but resistance to sulphonamides and penicillin occurs frequently. Resistance to erythromycin, fusidic acid, and gentamicin varies with the use of these drugs and the adequacy of control of cross infection; in Britain it occurs in less than $5 \%$ of strains. ${ }^{23}$ Multiply resistant strains occur sporadically, but recently "methicillin-resistant Staph aureus" strains have caused epidemics of infection in hospitals in Australia, ${ }^{+}$ North America, ${ }^{5}$ and Europe, including several in London. ${ }^{6}$

In contrast, haemolytic streptococci of Lancefield's group A remain highly sensitive to the penicillins, ${ }^{7}$ as are streptococci of groups B, ${ }^{8} \mathrm{C}$, and $\mathrm{G} .{ }^{9}$ Resistance to erythromycin is infrequent among these organisms, 'but it is associated with $M$ types 4 and 12 of group A streptococci. ${ }^{10}$ " Rapid increases in resistance to erythromycin were recorded between 1971 and 1974 in some areas of Japan ${ }^{12}$ and Canada ${ }^{13}$ in group A streptococci, but a rate of $0.3 \%$ was recently reported in Britain. ${ }^{10}$ Resistance to sulphonamides and tetracyclines by $\beta$ haemolytic streptococci is substantial.

Among the drugs used against anaerobic bacteria metronidazole continues to be active against bacteroides and other obligate anaerobes. Bacteroides fragilis is widely resistant to the penicillins, occasionally to clindamycin, and rarely to chloramphenicol. ${ }^{14} 15$

Multiply resistant strains of Streptococcus pneumoniae and Haemophilus influenzae, common pathogens of the respiratory tract and of meningitis, have been isolated in several countries but are not yet a practical problem.16 ${ }^{17}$ For pneumococci penicillin remains the drug of choice, but sensitivity must be confirmed because intermediate levels of resistance appear to be increasing. ${ }^{18}{ }^{19}$ Most strains are sensitive to erythromycin, chloramphenicol, and co-trimoxazole, ${ }^{16}{ }^{19}$ but substantial resistance may be found to tetracyclines, varying in place and time. ${ }^{20}$ At present some $5-22 \%$ of type B strains of $H$ influenzae resist ampicillin and its esters, usually by producing a $\beta$ lactamase probably acquired from Escherichia coli. ${ }^{21} \mathrm{Up}$ to $1 \%$ of non-capsulate strains are resistant to chloramphenicol ${ }^{21}$ and a very few, so far, to both agents. ${ }^{22} 23$ Resistance to other antibiotics is variably reported, with rates of nil to $80 \%$ for erythromycin, less for tetracyclines, ${ }^{24}$ and a low but increasing resistance to trimethoprim. ${ }^{25}$ Legionella pneumophila and Mycoplasma pneumoniae continue to be susceptible to erythromycin and the latter organism also to tetracyclines.

In meningitis outside the neonatal period the three common pathogens are $H$ influenzae, Str pneumoniae, and Neisseria meningitidis. The last has shown no change in sensitivity to benzylpenicillin, but the report of a strain able to produce $\beta$ lactamase through plasmids similar to those in $N$ gonorrhoeae may foretell the future. ${ }^{26}$ Increasing resistance to sulphonamides now prevents the use of these drugs in initial treatment. In endocarditis the different varieties of Str viridans continue to be sensitive to the penicillins, and the enterococci to amoxycillin. ${ }^{27}$ High level resistance by enterococci to gentamicin is increasing ${ }^{28}$ preventing synergy with penicillin..$^{29}$

Gram negative bacilli causing urinary tract infection outside hospital have shown little change in sensitivities in recent years, except for increasing resistance to amoxycillin ${ }^{30}$ and trimethoprim. ${ }^{31}$ In urinary infection acquired in hospital resistance occurs more often, owing to the selective pressure of antibiotics, ${ }^{30}$ and outbreaks with multiply resistant organisms are not unusual. ${ }^{22}$ Septicaemia and other infections caused by Gram negative bacilli, though more frequent, do not seem to be complicated by increasing resistance.

Of the bacteria causing gastrointestinal infections, Shigella sonne $i$ has shown some reduction in resistance in Britain from previous high levels, while other species of shigella more common abroad have shown increased resistance. ${ }^{33}$ Multiply resistant strains of Salmonella typhimurium are more frequent owing to the dissemination of particular phage types from poultry and cattle to man, ${ }^{34}$ and among other salmonellas resistance may be increasing. ${ }^{35}$ Campylobacters, now the most common cause of bacterial gastroenteritis, remain sensitive to erythromycin.

Penicillin resistant strains of $N$ gonorrhoeae continue to increase, reaching $1.6 \%$ of strains in Britain in $1982^{36}$; four of these strains were also resistant to spectinomycin. Cephalospurins and aminoglycosides are usually active against penicillin resistant strains. Chlamydiae, an important cause of non-specific genital infection, are susceptible to erythromycin and tetracycline.

What clinical strategies may be used to combat antibiotic resistance? Resistance is disseminated mainly by the exchange of genes between bacteria through plasmids ${ }^{37}$ and by the spread of resistant bacteria between patients both in hospitals and in the community. There is no direct way to prevent genetic exchange, but reducing the selective pressure exerted by antibiotics may help. Efficient antimicrobial treatment should aim at eliminating the pathogen quickly and expose the normal flora and the pathogen to antibiotics as little as possible. Inappropriate prescriptions, inadequate doses, topical antibiotics, and unjustifiably prolonged 